\title{
Genes targeted by the Hedgehog-signaling pathway can be regulated by Estrogen related receptor $\beta$
}

Yuan Lu ${ }^{1,2,5}$, Jilong Li ${ }^{2,3,4}$, Jianlin Cheng ${ }^{2,3,4}$ and Dennis B. Lubahn 1,2*

\begin{abstract}
Background: Nuclear receptor family member, Estrogen related receptor $\beta$, and the Hedgehog signal transduction pathway are both reported to relate to tumorigenesis and induced pluripotent stem cell reprogramming. We hypothesize that Estrogen related receptor $\beta$ can modulate the Hedgehog signaling pathway and affect Hedgehog driven downstream gene expression.
\end{abstract}

Results: We established an estrogen related receptor $\beta$-expressing Hedgehog-responsive NIH3T3 cell line by Esrrb transfection, and performed mRNA profiling using RNA-Seq after Hedgehog ligand conditioned medium treatment. Esrrb expression altered 171 genes, while Hedgehog signaling activation alone altered 339 genes. Additionally, estrogen related receptor $\beta$ expression in combination with Hedgehog signaling activation affects a group of 109 Hedgehog responsive mRNAs, including Hsd11b1, Ogn, Smoc2, Igf1, Pdcd4, Igfbp4, Stmn1, Hp, Hoxd8, Top2a, Tubb4b, Sfrp2, Saa3, Prl2c3 and Dpt.

Conclusions: We conclude that Estrogen related receptor $\beta$ is capable of interacting with Hh-signaling downstream targets. Our results suggest a new level of regulation of Hedgehog signaling by Estrogen related receptor $\beta$, and indicate modulation of Estrogen related receptor $\beta$ can be a new strategy to regulate various functions driven by the Hedgehog signaling pathway.

Keywords: Estrogen related receptor, Hedgehog signaling pathway, Gene expression profiling, RNA-Seq, Gene interactions

\section{Background}

Hedgehog (Hh) signaling is a pivotal signaling pathway in embryonic pattern formation, stem cell/cancer stem cell self-renewal, as well as induced pluripotent stem cells induction [1-12]. An early study showed the Hh-signaling inhibitor, cyclopamine, is enriched in Veratrum californicum. This plant when consumed by pregnant sheep resulted in a midline differentiation defect in offspring [13-17]. Hh-signaling activation can lead to reprogramming by driving the expression of Bmi1, and the endogenous Smoothened activator, oxysterol, can facilitate reprogramming [8].

\footnotetext{
*Correspondence: lubahnd@missouri.edu

1 Department of Biochemistry, University of Missouri, Columbia, MO 65211, USA

Full list of author information is available at the end of the article
}

Similar to other core development related pathways, deregulated Hh-signaling due to the mutation or overexpression of pathway components and/or pathway ligand induces a variety types of cancers including basal cell carcinoma, medulloblastoma, bladder cancer, breast cancer, cervical cancer, liver cancer, colon cancer, prostate cancer, gastric cancer, pancreas cancer, head and neck cancer, lymphoma and non-small cell lung cancer [18-32]. The pivotal role of Hh-signaling in cancer development makes Hh-signaling an attractive target for drug development [33-36]. For example, the FDA in 2012 approved GDC-0449, an Hh pathway inhibitor targeting Smoothened for basal cell carcinoma treatment [33, 37, 38].

Hh signaling pathway is controlled by membrane proteins Patched (Ptch) and Smo. When there is no ligand bound to Ptch, Ptch inhibits Smo and keeps the 
downstream pathway inactivated. When Ptch binds to Hh ligand, the inhibition of Ptch on Smo is relieved and the Hh-signaling pathway is activated. One of the broadly accepted mechanisms of Hh-signaling target genes response is through the binding of Gli family transcription factors to Gli-binding sites in the regulatory sequence of $\mathrm{Hh}$ regulated genes.

Esrrb belongs to the nuclear receptor family [39-41]. It is important in early embryo development as genomic knock out of Esrrb is embryonic lethal due to the placenta deformation resulted from early differentiation of trophoblast stem cells [42]. Recent research showed that Esrrb was found to be a core reprogramming factor in inducing pluripotent stem cells (iPSC). C-myc and klf4 of Yamanaka factors (Oct4, Sox2, Klf4, c-Myc) can be replaced by Esrrb [43-46]. Esrrb was also reported to drive Sox 2 transcription to induce reprogramming in a single cell reprogramming system, revealing its central role in differentiation [47]. In addition, Esrrb was found to play an important role in tumorigenesis in both in vitro and in vivo studies. It is down-regulated in prostate cancer and re-expression of Esrrb in prostate cancer cells inhibited cancer cell proliferation through tumor suppressor Cdkn1a/p21 induction [48, 49].

Esrrb has been reported to be constitutively active in the absence of a ligand and this is supported by the evidence that Esrrg, which shares over $80 \%$ of its Ligand Binding Domain with Esrrb, has a transcriptional active conformation similar to E2-activated Estrogen Receptor [50-53]. Another explanation for this endogenous activity is that Esrrb binds to an unknown endogenous ligand. The Esrrb endogenous ligand hypothesis is supported by a report that culturing the cells with charcoal-stripped serum-containing medium can eliminate the transcriptional activity of Esrrb on SFRE/ERRE [54].

Knocking down Esrrb in mouse embryonic stem cells was shown to affect the transcription of several Hh-signaling pathway related genes, including Gli2 and several Wnt family members, indicating Esrrb can potentially regulate Hh driven gene expression [55].

Although Gli transcription factors are relatively well known for transmitting Hh-signaling to target genes, other factors mediating the Hh-signaling activity are not well studied. For example, Dner, Fbn2, Hsd11b1 and Brak are Hh responsive genes in fetal prostate, but overexpression of Gli1 or Gli2 cannot affect the transcription of these 4 genes. However, the expression of active Smo significantly increased the mRNA concentration of these genes [56], indicating there is at least one other mechanism accounting for the Hh-signaling target gene transcription regulation.

Due to the importance of both Esrrb and Hh-signaling in development and tumorigenesis, we hypothesized that
Esrrb can regulate Hh-mediated transcription regulation and can serve as a regulator of Hh-signaling target genes. By employing mRNA profiling, we emphasized on the discovery of Esrrb-regulated Hh-signaling pathwaytargeted genes and we report 109 genes that differentially respond to Hh-signaling activation with Esrrb present.

\section{Results}

\section{Establishment of model cell lines}

With the purpose of exploring whether Esrrb can regulate Hh-signaling targeted genes, we require a Hh-responsive cell line. NIH3T3 cells are commonly used as Hh-responsive cell line. Additionally, this cell line does not express Esrrb, making it a good model that provides clean background of Esrrb. To make the NIH3T3 cells Esrrb positive, we stably transfected Esrrb into NIH3T3 cells. The control vector transfected NIH3T3 cells (NIH3T3-pc3.1) have no Esrrb expressed as mentioned before. In contrast, NIH3T3 cells transfected with Esrrb expression vector (NIH3T3-Esrrb) have significantly increased Esrrb protein concentration (Fig. 1a). Compared to HEK293 cells, which have endogenous Esrrb expressed but lack of detectable Hh-signaling response, the concentration of overexpressed Esrrb in NIH3T3 cells attained a physiological relevant concentration (Fig. 1a).

\section{Hedgehog signaling and Esrrb regulated genes}

To comprehensively characterize Hh-signaling driving mRNA changes in our system, we performed RNA-Seq analysis on the mRNA isolated from NIH3T3-pc3.1 cells treated with vehicle control or $1 \%$ Hh ligand conditioned medium (Hh-CM). Hedgehog signaling pathway activation after Hh-CM treatment was confirmed by significantly increased concentration of Gli1 by westernblot (Fig. 1b). After the Hh-CM treatment, we distinguished a total of 339 (245 up-regulated, 94 down-regulated) altered mRNAs (Figs. 2, 3a, Additional file 1: Table S3). We collected published Hh-signaling target gene sets generated by activating the Hh-signaling pathway or Gli transcription factor overexpression/knock-down in different model cell lines or tissues as Ref. [56-63]. For all of the non-redundant 1348

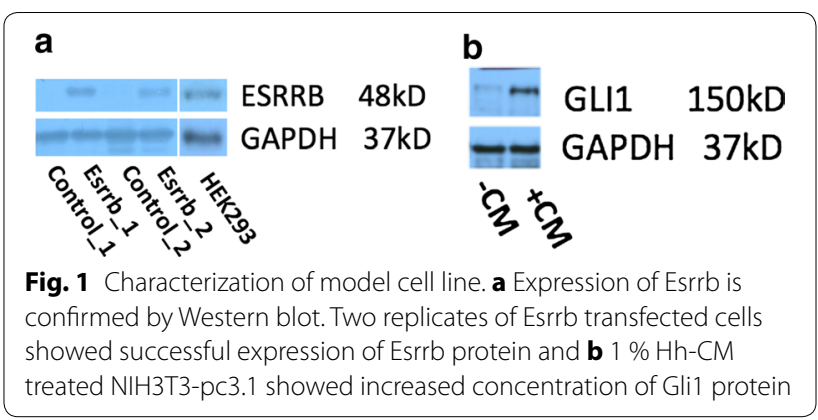




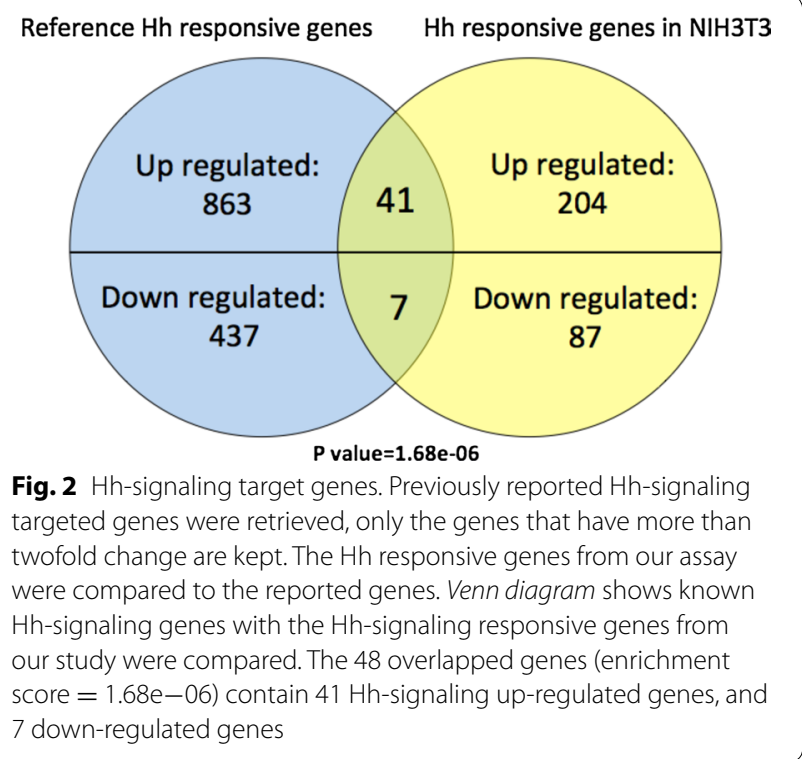

genes from previous reports, we found 48 genes (enrichment $p$ value $=1.68 \mathrm{e}-06$ ) that overlapped and another 291 new Hh-signaling responsive genes (Fig. 2).

We also surveyed the gene expression in NIH3T3Esrrb cells. 171 genes were differentially expressed by Esrrb expression (Additional file 1: Table S2; Additional file 2: Figure S1). We compared Esrrb-regulated genes to Hh-induced genes, and found there are 12 genes, Fabp4, Phex, Ccl5, Tagln, Aldh1a7, Lmod1, Cesla, Igf1, Mafb, Steap4, Pfkfb3 and Hlf, which are up-regulated or downregulated by either Hh ligand or Esrrb expression (Additional file 2: Figures S1, S2, \#1-8). The presence of these genes implies that Hh-signaling and Esrrb have functional overlaps in the regulation of these genes.

\section{Hh-signaling regulated genes in the presence of Esrrb}

Next we treated NIH3T3-Esrrb cells with Hh-CM to test gene response. Esrrb expression with Hh-CM treatment led to the largest amount of altered mRNAs (Fig. 3a). Supportively, spearman ranking correlation of all mRNA profiles in four different conditions showed that each condition generates different mRNA profiles, and correlation between Hh-signaling pathway stimulation in the presence of Esrrb expression and the no Esrrb no Hh stimulation control resulted in the lowest correlation coefficient in all pairwise comparisons (Fig. 3b).

Theoretically, mRNA profiling from four different conditions (Control, Hh-CM, Esrrb, Esrrb + CM) generates six different differentially expression pairwise comparisons. Since the comparison "Hh-CM vs. Esrrb" lacks of apparent interest, only five comparisons were left (refer to "Methods"). Every gene can be differentially expressed or not in any of the five given comparisons, therefore the comparison results can contain $2^{5}$ different possibilities for each gene.

We defined a group of genes as "Hh differentially response genes" as they response to Hh-CM when there is no Esrrb, while when Esrrb is expressed, their response to $\mathrm{Hh}-\mathrm{CM}$ is further enhanced, depressed or lost. These genes will indicate Esrrb can regulate Hh-signaling pathway activity. To find Hh differentially response genes, we used computer assisted gene sorting ("Methods", Additional file 2: Figure S2). The genes that fit our definition of "Hh differentially response" were found in groups \#1, \#2, \#3, \#4, \#5, \#9, \#10 and \#14 (Additional file 1: Table S3). Using the indicated filters of fold change and RPKM value described in "Methods", we classified 109 genes that differentially respond to $\mathrm{Hh}-\mathrm{CM}$ treatment when Esrrb is expressed (Additional file 2: Figure S2). We confirmed the concentration of 15 highly expressed mRNAs (saa3, prl2c3, dpt, sfrp2, pdcd4, smoc2, igf1, stmn1, top $2 a$, tubb4b, hp, hoxd8, igfbp4, hsd11b1, ogn) by qPCR. Pearson correlation coefficients between RNA-Seq and qPCR are at least 0.9 (Additional file 2: Figure S4). Among tested mRNAs, we found that when Esrrb expressing cells are treated with Hh-CM, Sfrp2 (secreted frizzle related protein 2), Saa3 (serum amyloid A3), Prl2c3 (prolactin 2A3), Stmn1 (stathmin1), Hp (haptoglobin), Hoxd8 (homeoboxD8), Tubb4b (tubulin beta 4B), Top $2 a$ (topoisomerase II alpha) and Dpt (dermatopontin) had different mRNA concentrations compared to Hh-CM treatment in cells without Esrrb. These differences are more likely due to a proportional additive effect of Esrrb expression on altered baseline expression of the mRNAs (Fig. 4a). In contrast, Igf1 (Insulin-like growth factor 1), Pdcd4 (programmed cell death 4) and Smoc2 (SPARC related calcium binding 2) lost their response to $\mathrm{Hh}$ stimuli when Esrrb was present (Fig. 4b). On the other hand, Hsd11b1 (hydoxysteroid 11 beta dehydrogenase 1), Igfbp4 (insulin-like growth factor binding protein 4) and $O g n$ (osteoglycin) responded to Hh-CM better when Esrrb was expressed (Fig. 4b).

\section{Discussion}

We distinguished 339 Hh-signaling altered mRNAs, among which are 48 known Hh-signaling target genes and 291 newly discovered targets (Fig. 3a, Additional file 1: Table S2). Most importantly, we characterized 109 genes that behave differently in response to Hh ligand in the presence versus the absence of Esrrb expression. This group of genes contains genes as Igfl, which respond to Hh ligand treatment in the absence of Esrrb, but when Esrrb is expressed, Hh ligand treatment cannot modify its transcription (Fig. 4b); genes as Hoxd8, which has differential expression in response to $\mathrm{Hh}$ stimulation 


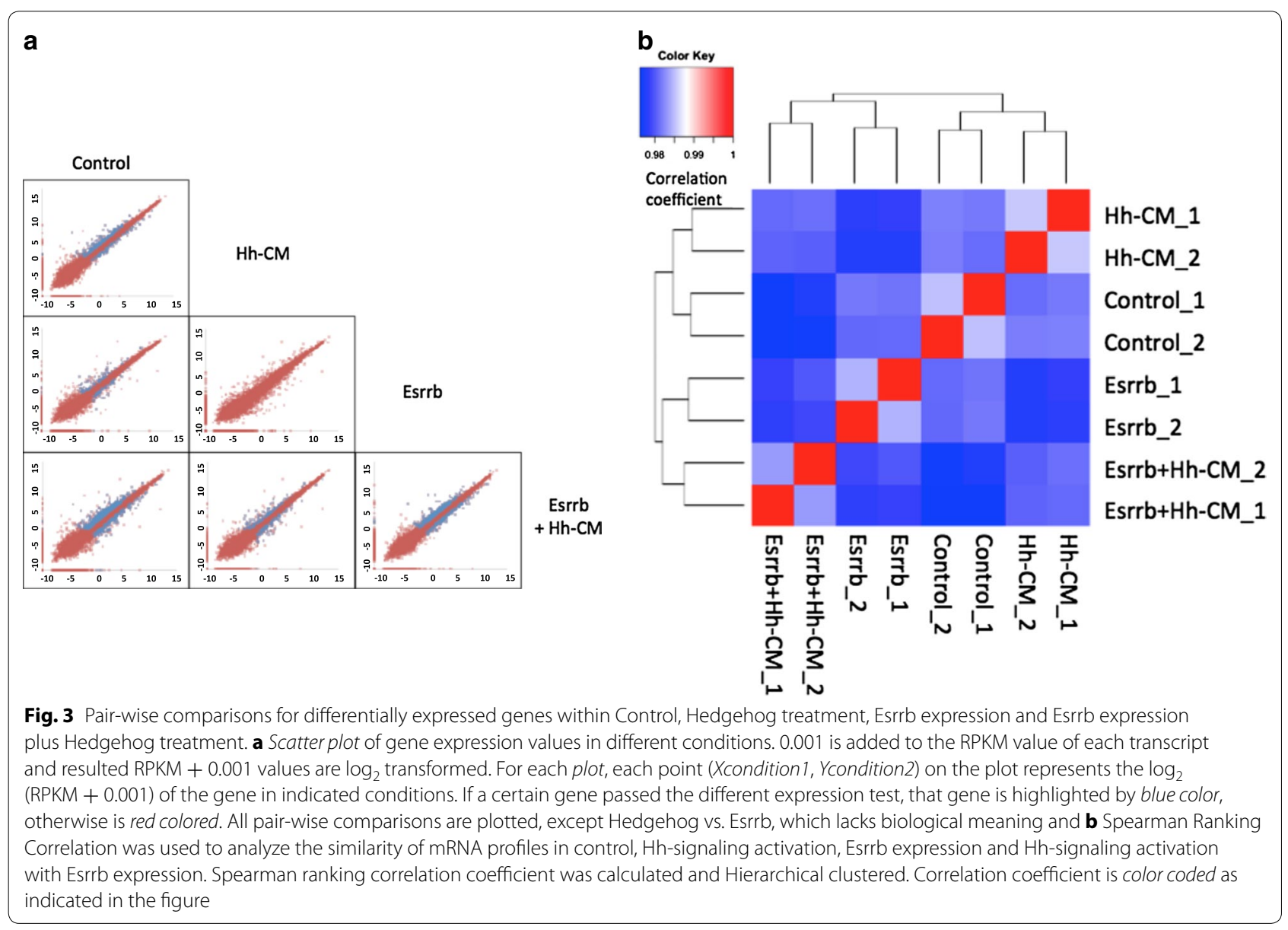

when Esrrb is expressed, though this different response comes from the effect of Esrrb on basal level mRNA concentration (Fig. 4a). We also observed genes like Hsd11b1 (Hh-CM vs. control: 21-fold; Esrrb + Hh-CM vs. Esrrb: 59 fold), Igfbp4 (Hh-CM vs. control: 3.4-fold; Esrrb + Hh-CM vs. Esrrb: 7.6 fold), and Ogn (Hh-CM vs. control: 57 \% inhibition; Esrrb + Hh-CM vs. Esrrb, $83 \%$ inhibition), which indicate that Esrrb and the Hh-signaling pathway synergistically regulate these genes (Fig. 4b). These results demonstrated that Esrrb is capable of interacting with Hh-signaling pathway and potentially regulate Hh-signaling downstream targets.

In the canonical Hh-signaling pathway model, the activation of Smo transmits the Hh signal to Gli transcription factor through the activation and inactivation of several pathway components including Fused [30] and Suppressor of Fused (SuFu). However, the evidence that the transcription of $H s d 11 b 1, F b n 2$ and Brak respond to active Smo transfection, but not Gli1 or Gli2 overexpression, indicates that Smo activation has a Gli-independent function in regulating gene expression [56]. In support of this hypothesis, we listed all the transcription factors; chromatin remodeling factors and transcription co-factors that response to $\mathrm{Hh}$-signaling activation for future reference (Additional file 2: Figure S2).

There are 21 genes (group \#25) that respond to Hhsignaling but only when Esrrb is present $(\log 2 \mathrm{FC}>1$ or $\log 2 \mathrm{FC}<-1$ ) (Additional file 2: Figure S5). 20 of them are up-regulated and 1 is down-regulated. This indicates Esrrb has the ability to expand the transcription regulation of the Hh-signaling pathway.

Additionally, we found cortisol-cortisone converting enzyme Hsd11b1 is correlated to Hh-signaling activation from several reports in several model systems including fetal prostate, prostate cancer and embryonic fibroblast cell lines [56, 64], indicating cortisone converted from cortisol by Hsd11b1 may account for part of the Hh response gene profile. Surprisingly, we found the classic Glucocorticoid Receptor (GR) target gene, $M t 2$, along with $3 \mathrm{Hh}$-signaling activation inhibits all other genes that have Glucocorticoid Response Elements in their promoter regions, Aldh1a7, Ankrd1 and Ism1 are repressed in response to $\mathrm{Hh}$ treatment (Additional file 2: Figure S6). Although Esrrb does not change the expression 


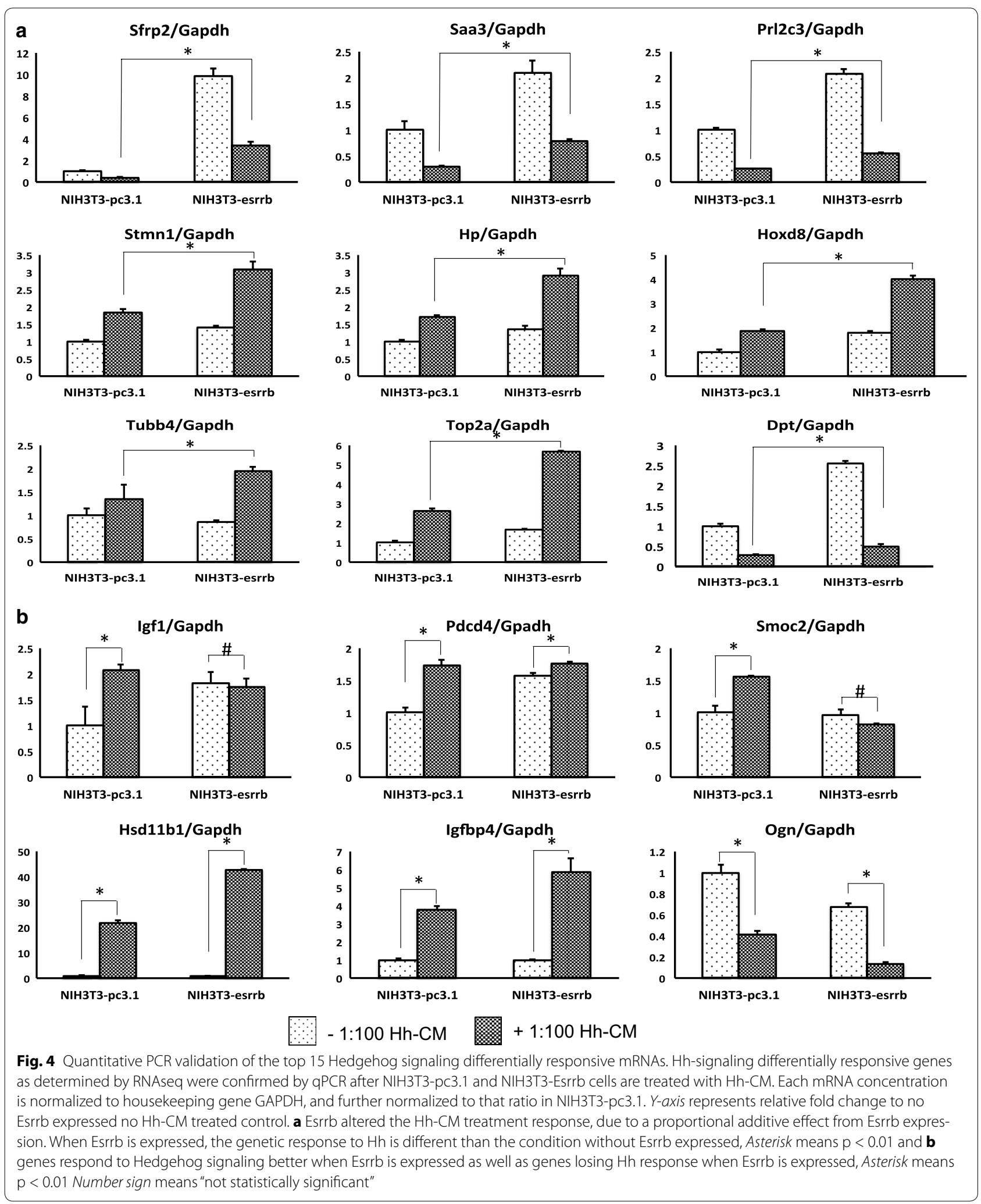


of Hsd11b1, Hh treatment with Esrrb expression further increased the mRNA concentration of Hsd11b1, accompanied by statistically significant alterations in concentrations of Mt2, Aldh1a7, Ankrd1 and Ism1 (Additional file 2: Figure S6). Our discovery strongly supports the idea that metabolite(s) downstream of Smo can also be mediators of Hh-signaling responses. Interestingly, GR overexpression and the activation of its target genes are strongly associated with anti-androgen treatment in prostate cancer therapy. GR target genes overlap with those of the Androgen Receptor and have been determined to be involved in antiandrogen treatment enzalutamide resistance [65]. Inhibiting GR can restore enzalutamide sensitivity. Esrrb's activity in increasing Hsd11b1 indirectly represses GR activity by potentially lowering the GR ligand cortisol, and thus activating Esrrb may lead to better response of antiandrogen treatment and eliminate or postpone the resistance.

\section{Conclusions}

Our data provided useful reference marker genes for both Hh-signaling and Esrrb function. In addition, we also showed that Esrrb has a role in the regulation of Hhsignaling driven genes. The mechanism of Hh-signaling was also expanded and a new layer of regulation of the Hh pathway through Esrrb was revealed, which may lead to improved treatments in Hh-signaling driven diseases.

\section{Methods}

\section{Conditional medium}

Sonic Hedgehog conditioned medium (Hh-CM) is collected from cultured HEK293 cells carrying Sonic Hedgehog (Shh) N-terminus transgene, which was a gift from Dr. Phillip Beachy's lab. Briefly, the Shh stabletransfected cells were maintained in Dulbecco's Modified Eagal Medium (DMEM, Invitrogen, Grand Island, NY, USA) with $10 \%$ Fetal Bovine Serum (FBS) until confluent, the medium was switched to DMEM with $0.2 \%$ FBS after 2 days. The medium enriched with Shh- $\mathrm{N}$ terminus was collected $24 \mathrm{~h}$ later, and was filtered through $0.22 \mu \mathrm{M}$ filter, and stored in $-80^{\circ}$ freezer [32].

\section{Cell lines and Expression vectors}

All cell lines used in this study were obtained commercially, and we did not conduct any animal work to obtain them. Paracrine Hh-responsive mouse embryonic fibroblast cells NIH3T3 were obtained from American Type Culture Collection (ATCC, Cat. No. CRL-1658). NIH3T3 cells are cultured in DMEM supplement with $10 \%$ Newborn Calf Serum (NBCS). The cells were transfected with pcDNA3.1 (Zeo+) empty vector (Promega, Madison, WI) as control, or pcDNA3.1 (Zeo+)-Esrrb expression vector using Fugene HD (Promega, Madison, WI), and were further selected by supplementing $150 \mu \mathrm{g} / \mathrm{ml}$ Zeocine (Invitrogen, Grand Island, NY) to the culture medium for 3 weeks. Two independent transfections were performed and established cells from each transfection were pooled together. Cells were cultured until confluent, and are treated with 1:100 diluted Hh-CM for $48 \mathrm{~h}$, in phenol-red free DMEM supplement with $5 \%$ NBCS. The Hh-CM treatment was controlled by the same dilution of medium cultured HEK293 cells that do not express Shh $\mathrm{N}$-terminus transgene.

\section{Reverse transcriptase PCR and real time PCR}

Total RNA was isolated and purified from two biological replicates of NIH3T3-pc3.1 and NIH3T3-Esrrb using RNeasy kit (Qiagen, Venlo, Netherlands) respectively. 1000 ng of total RNA was used to create cDNA libraries using Superscript III Reverse Transciptase (Invitrogen, Grand Island, NY) with random primers and oligodT. Quantitative PCR (qPCR) assays were carried out using SYBR GREEN iQ supermix (BioRad, Herculus, CA, USA) on ABI7500 system (Applied Biosytems, Foster City, CA, USA). Each qPCR assay was repeated 3 times. qPCR condition: $95^{\circ}, 30 \mathrm{~s} ; 60^{\circ}, 40 \mathrm{~s} ; 72^{\circ}, 40 \mathrm{~s}$. Primer sequences:

GAPDH (NM_008084), forward primer: AGCCTCGT CCCGTAGACAAAAT, reverse primer: CCGTGAGT GGAGTCATACTGGA;

Patched (NM_008957), forward primer: CTCTGGAG CAGATTTCCAAGG, reverse primer: TGCCGCAG TTCTTTTGAATG;

Gli1 (NM_010296), forward primer: GGAAGTCCT ATTCACGCCTTGA, reverse primer: CAACCTTCT TGCTCACACATGTAAG;

Igf1 (NM_001111274), forward primer: TGAGTGG CTTCCCTTGGGGG, reverse primer: AGGTGTTGT TTTGTGGGTGGGGT;

Smoc2 (NM_022315), forward primer: GGAAGGAG CAGGGAAAGCAGATGAT, reverse primer: TGGGCT GCTTGGCTTCCTCAAG;

Pdcd4 (NM_001168491), forward primer: GGACACT CCTAGGGCACCGC, reverse primer: TCCGCTTCC CGCCTTTGGAC;

Stmn1 (NM_019641), forward primer: TCGGACCGA GCAGGGCTTTC, reverse primer: CCGAGGGCT GAGAATCAGCTCAA;

Hp (NM_017370), forward primer: GAGGCAGTGTG TGGGAAGCCC, reverse primer: GGTCAGCAGCCAC TGGTCACT;

Ogn (NM_008760), forward primer: ACGACCTGGA ATCTGTGCCTCC, reverse primer: TTGGATTGCCCT CCAGGCGA;

Hoxd8 (NM_008276), forward primer: TTCCCTG GATGAGACCACAAGCAGC, reverse primer: GTCTC TCCGTGAGGGCCAGAGT 
Dpt (NM_019759), forward primer: TCAGTGCTGGA TCGTGAGTGGC, reverse primer: ACTGGCGATC CCTTTCCACTGC;

Top2a (NM_011623), forward primer: CCCAGGGAA GCTCCATGTCGG, reverse primer: GGTTCCCTTTGG CGCAGCTC;

Igfbp4 (NM_010517), forward primer: GATCGTGG GGACACCTCGGG, reverse primer: GCGGGGTGAC ACTGTTTGGGG;

Tubb4b (NM_146116), forward primer: TGTTGGCA GAGCGTCGGTTGT, reverse primer: CGCTGATTA CCTCCCAGAACTTGGC;

Hsd11b1 (NM_001044751): forward primer: CTGCCT GCCTGGGAGGTTGT, reverse primer: TCCCTGGAG CATTTCTGGTCTGAAC;

Sfrp2 (NM_009144): forward primer: GGCCACAGA GGAAGCTCCCAA, reverse primer: TCGGACACGCCG TTCAGCTT;

Saa3 (NM_011315): forward primer: ACAGCCAAAGA TGGGTCCAGTTCA, reverse primer: ACAGCCTCTCT GGCATCGCTGA;

Prl2c3 (NM_011118): forward primer: AGCCAGGC TCACACACTATGCAG, reverse primer: CCCGTTCCG GACTGCGTTGA;

\section{Immunoblot}

600,000 cells were plated in 6-well-plate. After $24 \mathrm{~h}$ growth in medium containing $10 \% \mathrm{NBCS}$, the cells were treated with $1 \% \mathrm{Hh}-\mathrm{CM}$ in phenol-red free DMEM with $5 \%$ NBCS for $48 \mathrm{~h}$. Cells were lysed by protein sample buffer (BioRad, Herculus, CA, USA) and boiled for $5 \mathrm{~min}$ at $95^{\circ} .20 \mu \mathrm{g}$ of total protein was loaded on $12 \%$ SDSPAGE gel. Electrophoresis was then performed. The proteins were then transferred to nitrocellulose membrane. The membrane was block by Phosphate buffered saline (PBS) with $0.05 \%$ Tween $20,0.015 \mathrm{~g} / \mathrm{ml}$ dry milk and $0.015 \mathrm{~g} / \mathrm{ml}$ bovine serum albumin (BSA). The membrane was then incubated with 1:2000 diluted monoclonal anti-Gli1 mouse IgG (Cell signaling, Beverly, MA, Cat.No: L42B10), 1:2000 diluted monoclonal anti-Esrrb mouse IgG (R\&D system, Cat. No: PP-H6705-00) and 1:2000 diluted polyclonal anti-GPADH rabbit IgG (Santa Cruz, Dallas, TX, Cat. No: sc-25777) overnight, washed, incubated with secondary antibodies diluted in PBS with $0.01 \mathrm{~g} / \mathrm{ml}$ BSA. The chemoluminescence was generated by west-Dura (Promega, Madison, WI, USA) and recorded by X-ray film (Fisher Scientific, Pittsburg, PA, USA).

\section{Deep sequencing and data analysis}

$2500 \mathrm{ng}$ total RNA from 2 biological replicates of each culture condition were extracted and purified, and then used to generate sequencing cDNA library using TruSeq
Stranded mRNA Sample Preparation kits (Illumina, San Diego, CA). Eight samples were pooled in one lane and each sample was ligated to one specific barcoded aligner. cDNA libraries quality was determined by University of Missouri DNA core. Deep sequencing was performed by University of Missouri DNA core using Illumina HiSeq 2000. Around 18 million reads were generated in.fastq format for each sample (raw data and data repository accession number will be available upon manuscript acceptance). The sequencing reads were trimmed and filtered using FASTX-Toolkit (http://hannonlab.cshl.edu/ fastx_toolkit), and mapped to UCSC mm9 genome using Bowtie2 and TopHat2 [66, 67]. Genome mapping results were submitted to Galaxy public server (galaxy.psu.edu) for gene expression value quantification. Gene expression value is represented as Reads Per Kilobase of transcript per Million mapped reads (RPKM) by Cufflink reference genome guided transcript assembly. Gene models from different experiment were merged together by Cuffmerge and all pair-wise comparisons of relative mRNA concentrations were analyzed by Cuffdiff. Differentially expressed genes are determined by False Discovery Rate adjusted p-value (q-value $<0.05$ ), $\log _{2}$ Fold Change $\left(\log _{2} \mathrm{FC} \geq 1\right.$ or $\left.\leq-1\right)$.

\section{Gene sorting and Hh differentially response genes characterization}

Five pairwise comparisons, (1) control vs. Hh-CM, (2) control vs. Esrrb, (3) control vs. Esrrb + Hh-CM, (4) Hh-CM vs. Esrrb + Hh-CM, (5) Esrrb vs. Esrrb + Hh-CM, but not Esrrb versus Hh-CM treatment, were made through differentially expressed gene analysis Cuffdiff, and test results are stored for each transcript [Yes $(Y): q<0.05$, statistically significantly different; or No $(\mathrm{N})$ : $q>0.05$, not significant]. Each gene is then sorted into 1 of the 32 groups based on each test result using an in-house $\mathrm{R}$ script (available upon request, Additional file 2: Figure S1). For each of the 16 groups of $\mathrm{Hh}$ responsive genes (control vs. Hh-CM, q < 0.05), a logic determination is made to filter out the groups that have pairwise comparison results against themselves (group \#6, \#7, \#8, \#12, \#13, \#15, \#16) or groups with no real world interest (\#11). Genes in the rest groups (\#1, $\# 2$, \#3, \#4, \#5, \#9, \#10 and \#14) are for further data filter and analysis. For the genes that passed the Hh-CM vs. Esrrb + Hh-CM test, we further filtered out genes that have $-0.5<[($ RPKM(Esrrb + Hh-CM $)-$ RPKMHh-CM)/RPKMHh-CM $]<0.5,-0.5<[(\mathrm{RPKM}(\mathrm{Hh}-$ $\mathrm{CM}) \quad-$ RPKMcontrol)/RPKMcontrol $]<0.5$, and $-5<[$ RPKM $($ Esrrb + Hh-CM $)-$ RPKMHh-CM $]<5$. For the genes that do not pass the Hh-CM vs. Esrrb $+\mathrm{Hh}-\mathrm{CM}$, the genes that have $-0.5<[(\mathrm{RPKM}(\mathrm{Hh}-$ $\mathrm{CM})-\operatorname{RPKM}($ control))/RPKMcontrol $]<0.5$, 
$[($ RPKM(Esrrb + Hh-CM $)-$ RPKMHh-CM $) /$ RPKMHh-CM $]>0.1$ or $<-0.1,-0.5<[($ RPKM(Esrrb) RPKM(control))/RPKMcontrol] $<0.5$ are filtered out. All genes that have passed the above tests are classified as Hh differentially response genes, and they respond to Hh-CM treatment differentially in the conditions with or without Esrrb expression.

\section{Gene ontology and KEGG pathway analysis}

DAVID bioinformatics source 6.7 is used for gene ontology (GO) analysis and KEGG pathway analysis [68, 69]. The gene names from certain pairwise comparison result were submitted to DAVID server (http://david.abcc.ncifcrf.gov) and GO analysis were performed for biological process (BP). Minimum counts were set as default value (two counts) and maximum EASE score ( $\mathrm{p}$ value) was set to 0.05 . Same parameter was used for KEGG pathway analysis.

\section{Hh-signaling related genes enrichment analysis}

Previous published Hh-signaling related gene sets generated by deep sequencing or microarray were retrieved (Refer to Additional file 1: Table S1 for specific experiment conditions and Hh-signaling targeted gene set descriptions). Briefly, only mRNAs altered more than twofold are included. Up-regulated and down-regulated mRNAs are sub-grouped and mRNA associated sequence identifications are all converted to gene symbols. Hh altered mRNAs in our gene set are compared to the previous reported mRNAs, and enrichment $p$ value is calculated as previously described [70].

\section{Statistical analysis}

Spearman Ranking Correlation is analyzed using $\mathrm{R}$ (version 3.0.2). RPKM value for each mRNA from both biological replicate in each condition is collected for correlation. The resulted pairwise correlation coefficients are stored in a matrix and Hierarchical clustering is created by R/Bioconductor (version 2.13) package Heatplus 2. Each Spearman correlation coefficient is color transformed for data visualization. qPCR experiments were repeated 3 times on two biological replicates. qPCR results were analyzed using t-test $(\mathrm{p}<0.01)$. Hh differentially response mRNA concentrations measured by RNAseq and qPCR are correlated using Pearson method in R. Briefly, for each tested mRNA, qPCR tested relative concentration is normalized by the concentration of internal control GAPDH, and further normalized to control condition. RNA-seq generated RPKM values are normalized to the value from control. Correlation is then performed between the above two sets of normalized expression values.

\section{Availability of supporting data}

The data sets supporting the results of this article are available in the NCBI-GEO repository, GSE71209 http://www.ncbi.nlm.nih.gov/geo/query/acc. cgi?acc $=$ GSE71209.

\section{Additional files}

Additional file 1. Table S1: Known Hh-signaling pathway target genes. Table S2: Result of all pairwise comparisons of differentially expressed genes. Table S3: Hh-signaling differentially responsive genes.

Additional file 2. Figure S1: Genes regulated by either Esrrb or Hhsignaling pathway. Figure S2: Decision tree model of gene sorting. Figure S3: Hh-responsive transcription-related genes. Figure S4: Correlation of qPCR and RNA-seq of top 15 Hh differentially responsive genes. Figure S5: Esrrb-dependent Hh-responsive genes. Figure S6: Glucocorticoid Receptor related genes in response to Hh-CM and Esrrb.

\section{Abbreviations}

Esrrb: Estrogen related receptor $\beta$; Hh: Hedgehog; Hh-CM: Hh ligand conditioned medium; iPSC: induced pluripotent stem cells; OSKM: Oct4, Sox2, KIf4, CMyc; FBS: fetal bovine serum; RT: reverse transcriptase; KEGG: kyoto encyclopedia of genes and genomes; GO: gene ontology; FC: fold change.

\section{Authors' contributions}

YL established the model cell line and performed RNA isolation, Esrrb expression status test, western blot, differentially expressed gene analysis, functional enrichment of gene sets by GO and KEGG pathway, and statistical analysis.

$Y L, J L, J C$ performed sequence alignment and generated the gene expression count table for RNA-seq data analysis. YL and DBL conceived of the study, participated in its design and drafted the manuscript. All authors read and approved the final manuscript.

\section{Author details}

${ }^{1}$ Department of Biochemistry, University of Missouri, Columbia, MO 65211 USA. ${ }^{2}$ MU Center for Botanical Interaction Studies, University of Missouri, Columbia, MO 65211, USA. ${ }^{3}$ Computer Science Department, University of Missouri, Columbia, MO 65211, USA. ${ }^{4}$ Informatics Institute, University of Missouri, Columbia, MO 65211, USA. ${ }^{5}$ Xiphophorus Genetic Stock Center, Texas State University, San Marcos, TX 78666, USA.

\section{Acknowledgements}

The author would like to thank Nicholas Starkey, Yufei Li and Debbie Blaisdell for helpful discussion. This publication or project was made possible in part by Grant Number P50AT006273 from the National Center for Complementary and Integrative Health (NCCIH), the Office of Dietary Supplements (ODS), and the National Cancer Institute ( $\mathrm{NCl}$ ). Its contents are solely the responsibility of the authors and do not necessarily represent the official views of the NCCAM, ODS, $\mathrm{NCl}$, or the National Institutes of Health.

\section{Competing interests}

The authors declare that they have no competing interests.

Received: 2 July 2015 Accepted: 6 November 2015

Published online: 23 November 2015

\section{References}

1. Litingtung $Y$, Lei L, Westphal $H$, Chiang C. Sonic Hedgehog is essential to foregut development. Nat Genet. 1998;20:58-61.

2. Lum L, Beachy PA. The Hedgehog response network: sensors, switches, and routers. Science. 2004;304:1755-9.

3. Jessell TM. Neuronal specification in the spinal cord: inductive signals and transcriptional codes. Nat Rev Genet. 2000;1:20-9. 
4. Zhang Y, Kalderon D. Hedgehog acts as a somatic stem cell factor in the Drosophila ovary. Nature. 2001;410:599-604.

5. Reya T, Morrison SJ, Clarke MF, Weissman IL. Stem cells, cancer, and cancer stem cells. Nature. 2001:414:105-11.

6. Machold R, Hayashi S, Rutlin M, Muzumdar MD, Nery S, et al. Sonic Hedgehog is required for progenitor cell maintenance in telencephalic stem cell niches. Neuron. 2003;39:937-50.

7. Lai K, Kaspar BK, Gage FH, Schaffer DV. Sonic Hedgehog regulates adult neural progenitor proliferation in vitro and in vivo. Nat Neurosci. 2003;6:21-7.

8. Moon JH, Heo JS, Kim JS, Jun EK, Lee JH, et al. Reprogramming fibroblasts into induced pluripotent stem cells with Bmi1. Cell Res. 2011;21:1305-15.

9. Ingham PW, McMahon AP. Hedgehog signaling in animal development: paradigms and principles. Genes Dev. 2001;15:3059-87.

10. Lamm ML, Catbagan WS, Laciak RJ, Barnett DH, Hebner CM, et al. Sonic Hedgehog activates mesenchymal Gli1 expression during prostate ductal bud formation. Dev Biol. 2002;249:349-66.

11. Freestone $\mathrm{SH}$, Marker P, Grace OC, Tomlinson DC, Cunha GR, et al. Sonic Hedgehog regulates prostatic growth and epithelial differentiation. Dev Biol. 2003;264:352-62

12. Berman DM, Desai N, Wang $X$, Karhadkar SS, Reynon M, et al. Roles for Hedgehog signaling in androgen production and prostate ductal morphogenesis. Dev Biol. 2004;267:387-98.

13. Zeiss CJ, Zarfoss MK, Johnson EE, Dubielzig RR. Ocular anomalies and holoprosencephaly in a lamb. Vet Ophthalmol. 2008;11:30-3.

14. Lipinski RJ, Dengler E, Kiehn M, Peterson RE, Bushman W. Identification and characterization of several dietary alkaloids as weak inhibitors of Hedgehog signaling. Toxicol Sci. 2007;100:456-63.

15. Lee ST, Panter KE, Gaffield W, Stegelmeier BL. Development of an enzyme-linked immunosorbent assay for the veratrum plant teratogens: cyclopamine and jervine. J Agric Food Chem. 2003;51:582-6.

16. Bale AE. Sheep, lilies and human genetics. Nature. 2000;406:944-5.

17. Keeler RF, Binns W. Chemical compounds of veratrum californicum related to congenital ovine cyclopian malformations: extraction of active material. Proc Soc Exp Biol Med. 1964;116:123-7.

18. Taipale J, Beachy PA. The Hedgehog and Wnt signalling pathways in cancer. Nature. 2001;411:349-54

19. Fei DL, Li H, Kozul CD, Black KE, Singh S, et al. Activation of Hedgehog signaling by the environmental toxicant arsenic may contribute to the etiology of arsenic-induced tumors. Cancer Res. 2010;70:1981-8.

20. Mechlin CW, Tanner MJ, Chen M, Buttyan R, Levin RM, et al. Gli2 expression and human bladder transitional carcinoma cell invasiveness. J Urol. 2010;184:344-51.

21. Hatsell S, Frost AR. Hedgehog signaling in mammary gland development and breast cancer. J Mammary Gland Biol Neoplasia. 2007;12:163-73.

22. Varnat F, Duquet A, Malerba M, Zbinden M, Mas C, et al. Human colon cancer epithelial cells harbour active HEDGEHOG-GLI signalling that is essential for tumour growth, recurrence, metastasis and stem cell survival and expansion. EMBO Mol Med. 2009;1:338-51.

23. Xuan $Y H$, Jung HS, Choi YL, Shin YK, Kim HJ, et al. Enhanced expression of Hedgehog signaling molecules in squamous cell carcinoma of uterine cervix and its precursor lesions. Mod Pathol. 2006;19:1139-47.

24. Ma X, Chen K, Huang S, Zhang X, Adegboyega PA, et al. Frequent activation of the Hedgehog pathway in advanced gastric adenocarcinomas. Carcinogenesis. 2005:26:1698-705.

25. Wu WK, Cho CH, Lee CW, Fan D, Wu K, et al. Dysregulation of cellular signaling in gastric cancer. Cancer Lett. 2010;295:144-53.

26. Chung $\mathrm{CH}$, Dignam JJ, Hammond ME, Klimowicz AC, Petrillo SK, et al. Glioma-associated oncogene family zinc finger 1 expression and metastasis in patients with head and neck squamous cell carcinoma treated with radiation therapy (RTOG 9003). J Clin Oncol. 2011;29:1326-34

27. Huang $S$, He J, Zhang $X$, Bian $Y$, Yang $L$, et al. Activation of the Hedgehog pathway in human hepatocellular carcinomas. Carcinogenesis. 2006;27:1334-40

28. Dierks C, Grbic J, Zirlik K, Beigi R, Englund NP, et al. Essential role of stromally induced Hedgehog signaling in B-cell malignancies. Nat Med. 2007;13:944-51.

29. Ferretti E, De Smaele E, Di Marcotullio L, Screpanti I, Gulino A. Hedgehog checkpoints in medulloblastoma: the chromosome 17p deletion paradigm. Trends Mol Med. 2005:11:537-45.
30. Rudin CM, Hann CL, Laterra J, Yauch RL, Callahan CA, et al. Treatment of medulloblastoma with Hedgehog pathway inhibitor GDC-0449. N Engl J Med. 2009;361:1173-8.

31. Morton JP, Mongeau ME, Klimstra DS, Morris JP, Lee YC, et al. Sonic Hedgehog acts at multiple stages during pancreatic tumorigenesis. Proc Natl Acad Sci USA. 2007;104:5103-8

32. Karhadkar SS, Bova GS, Abdallah N, Dhara S, Gardner D, et al. Hedgehog signalling in prostate regeneration, neoplasia and metastasis. Nature. 2004:431:707-12.

33. De Smaele E, Ferretti E, Gulino A. Vismodegib, a small-molecule inhibitor of the Hedgehog pathway for the treatment of advanced cancers. Curr Opin Investig Drugs. 2010;11:707-18.

34. Metcalfe C, Alicke B, Crow A, Lamoureux M, Dijkgraaf GJ, et al. PTEN loss mitigates the response of medulloblastoma to Hedgehog pathway inhibition. Cancer Res. 2013;73:7034-42.

35. Sarris EG, Syrigos KN, Saif MW. Novel agents and future prospects in the treatment of pancreatic adenocarcinoma. JOP. 2013;14:395-400.

36. Gonnissen A, Isebaert S, Haustermans K. Hedgehog signaling in prostate cancer and its therapeutic implication. Int J Mol Sci. 2013;14:13979-4007.

37. Ansell PJ, Espinosa-Nicholas C, Curran EM, Judy BM, Philips BJ, et al. In vitro and in vivo regulation of antioxidant response element-dependent gene expression by estrogens. Endocrinology. 2004;145:311-7.

38. Robarge KD, Brunton SA, Castanedo GM, Cui Y, Dina MS, et al. GDC-0449-a potent inhibitor of the Hedgehog pathway. Bioorg Med Chem Lett. 2009;19:5576-81.

39. Pettersson K, Svensson K, Mattsson R, Carlsson B, Ohlsson R, et al. Expression of a novel member of estrogen response element-binding nuclear receptors is restricted to the early stages of chorion formation during mouse embryogenesis. Mech Dev. 1996;54:211-23.

40. Giguere V, Yang N, Segui P, Evans RM. Identification of a new class of steroid hormone receptors. Nature. 1988;331:91-4.

41. Chen F, Zhang Q, McDonald T, Davidoff MJ, Bailey W, et al. Identification of two hERR2-related novel nuclear receptors utilizing bioinformatics and inverse PCR. Gene. 1999;228:101-9.

42. Luo J, Sladek R, Bader JA, Matthyssen A, Rossant J, et al. Placenta abnormalities in mouse embryos lacking the orphan nuclear receptor ERR-beta. Nature. 1997;388:778-82.

43. Feng B, Jiang J, Kraus P, Ng JH, Heng JC, et al. Reprogramming of fibroblasts into induced pluripotent stem cells with orphan nuclear receptor Esrrb. Nat Cell Biol. 2009:11:197-203.

44. Takahashi K, Okita K, Nakagawa M, Yamanaka S. Induction of pluripotent stem cells from fibroblast cultures. Nat Protoc. 2007:2:3081-9.

45. Takahashi K, Tanabe K, Ohnuki M, Narita M, Ichisaka T, et al. Induction of pluripotent stem cells from adult human fibroblasts by defined factors. Cell. 2007;131:861-72

46. Okita K, Ichisaka T, Yamanaka S. Generation of germline-competent induced pluripotent stem cells. Nature. 2007;448:313-7.

47. Buganim Y, Faddah DA, Cheng AW, Itskovich E, Markoulaki S, et al. Singlecell expression analyses during cellular reprogramming reveal an early stochastic and a late hierarchic phase. Cell. 2012;150:1209-22.

48. Yu S, Wong YC, Wang XH, Ling MT, Ng CF, et al. Orphan nuclear receptor estrogen-related receptor-beta suppresses in vitro and in vivo growth of prostate cancer cells via p21(WAF1/CIP1) induction and as a potential therapeutic target in prostate cancer. Oncogene. 2008;27:3313-28.

49. Castet A, Herledan A, Bonnet S, Jalaguier S, Vanacker JM, et al. Receptorinteracting protein 140 differentially regulates estrogen receptor-related receptor transactivation depending on target genes. Mol Endocrinol. 2006;20:1035-47

50. Greschik H, Wurtz JM, Sanglier S, Bourguet W, van Dorsselaer A, et al. Structural and functional evidence for ligand-independent transcriptional activation by the estrogen-related receptor 3. Mol Cell. 2002;9:303-13.

51. Nam K, Marshall P, Wolf RM, Cornell W. Simulation of the different biological activities of diethylstilbestrol (DES) on estrogen receptor alpha and estrogen-related receptor gamma. Biopolymers. 2003:68:130-8.

52. Greschik H, Flaig R, Renaud JP, Moras D. Structural basis for the deactivation of the estrogen-related receptor gamma by diethylstilbestrol or 4-hydroxytamoxifen and determinants of selectivity. J Biol Chem. 2004;279:33639-46.

53. Abad MC, Askari H, O'Neill J, Klinger AL, Milligan C, et al. Structural determination of estrogen-related receptor gamma in the presence of phenol derivative compounds. J Steroid Biochem Mol Biol. 2008;108:44-54. 
54. Vanacker JM, Bonnelye E, Chopin-Delannoy S, Delmarre C, Cavailles $V$, et al. Transcriptional activities of the orphan nuclear receptor ERR alpha (estrogen receptor-related receptor-alpha). Mol Endocrinol. 1999;13:764-73.

55. Nishiyama A, Sharov AA, Piao Y, Amano M, Amano T, et al. Systematic repression of transcription factors reveals limited patterns of gene expression changes in ES cells. Sci Rep. 2013;3:1390.

56. Yu M, Gipp J, Yoon JW, lannaccone P, Walterhouse D, et al. Sonic Hedgehog-responsive genes in the fetal prostate. J Biol Chem. 2009;284:5620-9.

57. Xu X, Zhou Y, Xie C, Wei SM, Gan H, et al. Genome-wide screening reveals an EMT molecular network mediated by Sonic Hedgehog-Gli1 signaling in pancreatic cancer cells. PLoS One. 2012;7:e43119.

58. Lee JM, Miyazawa S, Shin JO, Kwon HJ, Kang DW, et al. Shh signaling is essential for rugae morphogenesis in mice. Histochem Cell Biol. 2011;136:663-75.

59. Mazumdar T, DeVecchio J, Agyeman A, Shi T, Houghton JA. The GLI genes as the molecular switch in disrupting Hedgehog signaling in colon cancer. Oncotarget. 2011;2:638-45.

60. Franco HL, Lee KY, Broaddus RR, White LD, Lanske B, et al. Ablation of Indian Hedgehog in the murine uterus results in decreased cell cycle progression, aberrant epidermal growth factor signaling, and increased estrogen signaling. Biol Reprod. 2010;82:783-90.

61. Narita S, So A, Ettinger S, Hayashi N, Muramaki M, et al. GLI2 knockdown using an antisense oligonucleotide induces apoptosis and chemosensitizes cells to paclitaxel in androgen-independent prostate cancer. Clin Cancer Res. 2008;14:5769-77.
62. Feldmann G, Habbe N, Dhara S, Bisht S, Alvarez H, et al. Hedgehog inhibition prolongs survival in a genetically engineered mouse model of pancreatic cancer. Gut. 2008;57:1420-30.

63. Hochman E, Castiel A, Jacob-Hirsch J, Amariglio N, Izraeli S. Molecular pathways regulating pro-migratory effects of Hedgehog signaling. J Biol Chem. 2006;281:33860-70

64. Mori R, Xiong S, Wang Q, Tarabolous C, Shimada H, et al. Gene profiling and pathway analysis of neuroendocrine transdifferentiated prostate cancer cells. Prostate. 2009;69:12-23.

65. Arora VK, Schenkein E, Murali R, Subudhi SK, Wongvipat J, et al. Glucocorticoid receptor confers resistance to antiandrogens by bypassing androgen receptor blockade. Cell. 2013;155:1309-22.

66. Langmead B, Trapnell C, Pop M, Salzberg SL. Ultrafast and memoryefficient alignment of short DNA sequences to the human genome. Genome Biol. 2009;10:R25

67. Trapnell C, Pachter L, Salzberg SL. TopHat: discovering splice junctions with RNA-Seq. Bioinformatics. 2009;25:1105-11.

68. da Huang W, Sherman BT, Lempicki RA. Systematic and integrative analysis of large gene lists using DAVID bioinformatics resources. Nat Protoc. 2009:4:44-57.

69. da Huang W, Sherman BT, Lempicki RA. Bioinformatics enrichment tools: paths toward the comprehensive functional analysis of large gene lists. Nucleic Acids Res. 2009;37:1-13.

70. Zhou Q, Chipperfield H, Melton DA, Wong WH. A gene regulatory network in mouse embryonic stem cells. Proc Natl Acad Sci USA. 2007;104:16438-43.

\section{Submit your next manuscript to BioMed Central and take full advantage of:}

- Convenient online submission

- Thorough peer review

- No space constraints or color figure charges

- Immediate publication on acceptance

- Inclusion in PubMed, CAS, Scopus and Google Scholar

- Research which is freely available for redistribution

Submit your manuscript at

www.biomedcentral.com/submit

C Biomed Central 